\title{
Another Milestone
}

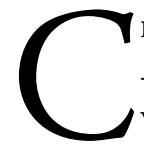

ELEBRATING DEVELOPMENTAL MILESTONES IS DEEPLY INGRAINED IN OUR CULTURE - witness the number of Facebook postings about a baby's first steps and first words. We would like to invite you to join in our celebration of this journal's most recent milestone: our 500th paper submission. Since the launch of Healthcare Policy/Politiques de Santé, we have received contributions on a very broad range of topics, from workforce planning, training and regulation to quality of care, sustainable funding and resource allocation, and governance and accountability. The best submissions appear in the journal's pages and are shared far and wide. Last year's best-read paper logged almost 2,500 views, with many more readers learning of key findings through the Longwoods eLetter, which currently has more than 11,000 opt-in subscribers.

In this issue, you will find evidence-informed commentary on a variety of topical issues, including medical tourism, the number of patients a family practice should have and the politics of access to expensive drugs. Our authors also share a range of new research results. The questions that they address are diverse, including how the media report technology assessment results, the experience with benchmarking in France and challenges with forecasting residential care requirements. Whatever your particular area of focus, I hope that you will find much of interest within this issue's pages.

Of course, our ability to edit and publish these and other submissions depends on a sound review process. As an editorial team, we wish to pay special tribute this month to all those who have volunteered their time over the past year to serve as peer reviewers for Healthcare Policy/Politiques de Santé. Our reviewers' careful observations and guidance are key to ensuring the quality and relevance of the papers that we publish. Approximately 70 percent of our reviewers come from Canada, with 30 per cent from other countries. A list of this year's reviewers appears on page 100. I would like to take this opportunity to thank all of them for their essential assistance.

Are you interested in becoming a reviewer? If so, please register for our database of potential reviewers at http //www.longwoods.com/reviewer-registration/healthcare-policy. This tool helps our editors match papers that we send out for comment with reviewers' expertise and interests. By registering, you can help advance scholarship and evidence-informed debate in health policy, both in the journal's pages and beyond. 


\section{Nouvelle étape franchie}

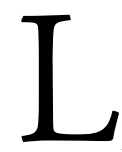

A CÉLÉBRATION DES ÉTAPES IMPORTANTES DU DÉVELOPPEMENT EST PROFONDÉMENT ancrée dans notre culture - il suffit de voir les nombreux affichages dans Facebook des premiers pas ou des premiers mots de bébé. Nous vous invitons à vous joindre à nous pour célébrer la plus récente étape de cette revue, soit la soumission du $500^{\text {e }}$ article. Depuis les débuts de Politiques de Santé/Healthcare Policy, nous avons reçu des articles sur une vaste gamme de sujets, par exemple la planification, la formation et la réglementation de la maind'œuvre, la qualité des soins, le financement durable, l'allocation des ressources, la gouvernance et la reddition de comptes. Les meilleures soumissions sont publiées dans les pages de la revue et leur contenu est abondamment partagé. L'année dernière, l'article le plus consulté a enregistré presque 2500 visites. Encore plus de lecteurs prennent également connaissances de résultats importants grâce au bulletin électronique de Longwoods, qui compte plus de 11000 abonnés.

Dans ce numéro, vous trouverez des commentaires éclairés par les données probantes sur plusieurs sujets, notamment le tourisme médical, le nombre de patients que devrait compter une clinique de médecine familiale ainsi que les politiques d'accès aux médicaments coûteux. Les auteurs partagent aussi de nombreux résultats tirés de nouvelles recherches. Ils abordent plusieurs questions comme la façon dont les médias font état des résultats d'évaluations des technologies de la santé, l'expérience du benchmarking en France et les défis liés aux prévisions des soins requis dans les établissements de longue durée. Quel que soit votre domaine, j’espère que vous trouverez un sujet de grand intérêt dans les pages de ce numéro.

Bien sûr, la publication de ces articles est tributaire d'un rigoureux processus de révision. En tant qu'équipe de rédaction, nous souhaitons rendre honneur à tous ceux qui ont donné leur temps au cours de l'année à titre d'évaluateurs pour Politiques de Santé/Healthcare Policy. Leurs observations judicieuses sont essentielles pour la qualité et la pertinence des articles que nous publions. Environ 70 pour cent de nos évaluateurs proviennent du Canada et 30 pour cent de l'étranger. La page 100 présente la liste des évaluateurs de cette année. J'aimerais profiter de cette occasion pour remercier chacun d'eux pour leur inestimable aide.

Être évaluateur vous intéresse? Si tel est le cas, veuillez vous inscrire dans notre base de données d'évaluateurs potentiels à http://www.longwoods.com/reviewer-registration/healthcare-policy. Cet outil aide nos éditeurs à jumeler les articles que nous envoyons pour révision à l'expérience et aux intérêts des évaluateurs. En vous y inscrivant, vous appuierez la science et les débats éclairés par les données probantes, tant dans les pages de la revue que dans le vaste milieu. 